\title{
Stimulus Reward Value Interacts with Training-induced Plasticity in Inhibitory Control
}

\author{
Michael De Pretto, ${ }^{\mathrm{a} \dagger}$ Lea Hartmann, ${ }^{\mathrm{a} \dagger}$ David Garcia-Burgos, ${ }^{\mathrm{b}}$ Etienne Sallard ${ }^{\mathrm{a}}$ and Lucas Spierer $^{\mathrm{a} *}$ \\ a Neurology Unit, Medicine Section, Faculty of Science and Medicine, University of Fribourg, Fribourg 1700, Switzerland \\ ${ }^{\mathrm{b}}$ Unit of Clinical Psychology and Psychotherapy, Department of Psychology, University of Fribourg, Fribourg 1700, Switzerland
}

\begin{abstract}
Training inhibitory control, the ability to suppress motor or cognitive processes, not only enhances inhibition processes, but also reduces the perceived value and behaviors toward the stimuli associated with the inhibition goals during the practice. While these findings suggest that inhibitory control training interacts with the aversive and reward systems, the underlying spatio-temporal brain mechanisms remain unclear. We used electrical neuroimaging analyses of event-related potentials to examine the plastic brain modulations induced by training healthy participants to inhibit their responses to rewarding (pleasant chocolate) versus aversive food pictures (unpleasant vegetables) with Go/NoGo tasks. Behaviorally, the training resulted in a larger improvement in the aversive than in the rewarding NoGo stimuli condition, suggesting that reward responses impede inhibitory control learning. The electrophysiological results also revealed an interaction between reward responses and inhibitory control plasticity: we observed different effects of practice on the rewarding vs. aversive NoGo stimuli at $\mathbf{2 0 0}$ ms post-stimulus onset, when the conflicts between automatic response tendency and task demands for response inhibition are processed. Electrical source analyses revealed that this effect was driven by an increase in right orbito-cingulate and a decrease in temporo-parietal activity to the rewarding NoGo stimuli and the reverse pattern to the aversive stimuli. Our collective results provide direct neurophysiological evidence for interactions between stimulus reward value and executive control training, and suggest that changes in the assessment of stimuli with repeated motoric inhibition likely follow from associative learning and behavior-stimulus conflicts reduction mechanisms. (c) 2019 IBRO. Published by Elsevier Ltd. All rights reserved.
\end{abstract}

Key words: inhibitory control, plasticity, training, food cues, ERP, source estimations.

\section{INTRODUCTION}

Training inhibitory control (IC), the ability to suppress cognitive or motor processes (Aron et al., 2004), not only reinforces the capacity to override impulsive reactions but also influences how the trained stimuli are assessed. Inhibitory control training has notably been shown to reduce the choice and the consumption of the trained NoGo stimuli (e.g. Houben and Jansen, 2011; Veling et al., 2013).

These 'collateral effects' of inhibitory control training may prove to be clinically relevant because they could help improving unhealthy eating habits or other types of

\footnotetext{
${ }^{*}$ Corresponding author. Address: Neurology Unit, Medicine Department, Faculty of Sciences, University of Fribourg, PER 09, Chemin du Musée 5, 1700 Fribourg, Switzerland.

E-mail address: lucas.spierer@unifr.ch (L. Spierer).

These authors contributed equally to this work.

Abbreviations: ACQ, Attitudes to Chocolate Questionnaire; BSI, Behavior-Stimulus interaction; EEG, electroencephalogram; ERP, event-related potential; IC, inhibitory control; IES, inverse efficiency score; LAURA, local autoregressive average; MNI, Montrea Neurological Institute; RS, Restraint Scale; RT, response times; RTt, response time threshold; SMAC, Spherical Model with Anatomical Constraints.
}

maladaptive reward-responses (Allom et al., 2016). For instance, Lawrence and colleagues (Lawrence et al., 2015a) showed that forty minutes of repeated inhibition of motor response to high energy density food reduces daily energy intake and participants' weight (for review, see e.g. Stice et al., 2016).

However, how repeated motoric inhibitions actually modify the neurocognitive processing of the food stimuli to eventually influence behavior toward them remains largely speculative; three main non-exclusive mechanisms have so far been advanced (Veling et al., 2017), which all predict specific patterns of traininginduced behavioral and electrophysiological plastic changes:

i) The training may first reinforce top-down inhibitory control processes, in turn helping participants to voluntarily resist impulses toward palatable food items. This mechanism predicts a modification of the brain responses to the NoGo stimuli during the implementation of the inhibition command, as indexed by the P3 ERP component 300 ms post-stimuli onset and 
within right ventrolateral prefrontal cortices (Manuel et al., 2010; Lenartowicz et al., 2011; Spierer et al., 2013; Berkman et al., 2014; Chavan et al., 2015; Hartmann et al., 2016; De Pretto et al., 2017). In addition, this account predicts larger behavioral improvements with appetitive than aversive pictures because higher rewarding value elicit stronger more difficult to inhibit- approach impulses.

ii) The IC training may also develop automatic associations between the food items used as the NoGo stimuli and the inhibition goal (for review, see Spierer et al., 2013). When stimulus-driven forms of inhibition develop, IC improves because the NoGo stimuli directly trigger behavioral inhibition or withdrawal, bypassing the slow voluntary initiation of the inhibition command. This mechanism predicts modifications during the discriminative/ attentional N1/P1 components at $150 \mathrm{~ms}$ poststimulus onset (Salinas and Stanford, 2013; Logan et al., 2014; Verbruggen et al., 2014b) and within the parietal areas implementing stimulus-response mapping rules (Manuel et al., 2010). In addition, this account predicts larger improvement for aversive than appetitive pictures as aversive NoGo stimuli are already associated with inhibition and withdrawal. A modification of the attentional biases to the stimuli may also manifest if IC training influences automatic responses to the stimuli (Goolsby et al., 2009; Kakoschke et al., 2015; Meule and Platte, 2016).

iii) As a third account, the Behavior Stimulus Interaction (BSI) theory suggests that IC training intervention may result in a devaluation of the food items, which would in turn influence behaviors toward them (Veling et al., 2008; Chen et al., 2016). At the beginning of the training, rewarding NoGo food stimuli elicit strong response tendencies conflicting with the task requirements for inhibiting motor responses. The pleasant food items would then be devaluated to reduce this action-related conflict (Chen et al., 2016). This mechanism mainly predicts modifications during the conflict detection/decisional N2 component at 200-300 ms (Falkenstein, 2006; Manuel et al., 2010; van de Laar et al., 2010; Verbruggen and Logan, 2015; Hartmann et al., 2016), and within anterior cingulate performance monitoring and orbitofrontal reward-related areas (Kringelbach and Rolls, 2004; Rolls and Grabenhorst, 2008). In addition, this account predicts better initial performance for the aversive than the rewarding pictures, and a larger increase in performance for the rewarding than the aversive pictures. Because of the tight link between stimulus value and saliency (e.g. Anderson et al., 2013), this account further predicts a decrease in the attentional bias to the rewarding but not aversive stimuli with training.

We tested these hypotheses using global, data-driven analyses of the ERP combined with distributed electrical source estimations recorded during Go/NoGo training tasks with either rewarding or aversive food picture as
NoGo stimuli (i.e. with opposite reinforcing value and action tendencies). We also measured attentional biases to the stimuli before and after the training with a Dot-probe task to further test the predictions of each of the models on attentional capture.

\section{EXPERIMENTAL PROCEDURES}

We recorded two groups of participants, one participating in the training with Rewarding and the other with Aversive food pictures as NoGo stimuli during the Go/NoGo training. Our data are available upon reasonable request to the corresponding author.

\section{Aversive group participants}

The 'Aversive' group included a total of 19 women. One participant was excluded from the analyses due to outlying behavioral performance (error rate above 2.5 SD from the group mean). A total of 18 participants were thus eventually included in the Aversive group (mean age $\pm S D=22.8 \pm 2.2$ years).

\section{Reward group participants}

The 'Reward' group included 21 women. Two of them were excluded from the analyses, one because of a lack of sleep and the other because she performed part of the task with two fingers at the same time. A total of 19 participants were thus eventually included in the Reward group (Mean age $\pm S D=22.8 \pm 2.3$ years).

Since we chose chocolate pictures as the rewarding stimuli (see the 'Stimulus' section), we further controlled several demographic and psychological parameters known to influence reward responses to food stimuli. We first controlled that the participants were within a healthy weight range (average body mass index of 21.5 $\pm 2.2 \mathrm{~kg} / \mathrm{m}^{2}$ (BMl: weight $\left.\left.(\mathrm{kg}) /[\text { height }(\mathrm{m})]^{2}\right)\right)$. In addition, to ensure that the chocolate pictures indeed had an appropriate rewarding value, participants were selected if they considered themselves as 'chocolate lovers', but without pathological attitudes toward chocolate (ACQ; Attitudes to Chocolate Questionnaire; Benton et al., 1998; total score $=4.1 \pm 1.3$; Craving score $=4.2$ $\pm 1.8)$. We also verified that they did not have any dietary restrictions (Restraint Scale (RS); Herman and Polivy, $1980 ; R S=13.6 \pm 3.2$ ).

\section{General criteria for participants' selection}

In addition to these group-specific criteria, we ensured for both groups that they i) did not have any current states of anxiety and depression (HADS; Zigmond and Snaith, 1983; Anxiety $=8.5 \pm 4.4 ; \quad$ Depression $=4.7 \pm 3.1$ ), and ii) were free of medical treatment, without history of diagnosed psychiatric or neurologic disorders, and had normal or corrected-to-normal vision. Since hunger has been shown to influence IC and the way food-stimuli are processed (Nederkoorn et al., 2009; Nijs et al., 2010; Loeber et al., 2013; Meule et al., 2014), we also controlled hunger levels by instructing participants to eat two hours before the experiment and by measuring participants' 
current level of hunger (Visual Analogue Scale for hunger; Reward group: $1.5 \pm 1.7$; Aversive group: $2.6 \pm 2.1$ ) Furthermore, given that IC deficits and impulsivity are positively associated with several facets of unhealthy eating (Jasinska et al., 2012) we also controlled impulsivity (BIS-11; Barratt Impulsiveness Scale; Patton et al., 1995; $\mathrm{BIS}=60.2 \pm 6.6$ ).

\section{Stimuli}

For the Rewarding group, we selected five chocolate (rewarding NoGo stimuli) and twenty non-chocolate digital colored photographs (Go stimuli; e.g. flower, book, or butterfly) from a picture database (Blechert et al., 2014). We used pictures of chocolate as rewarding stimuli because of $i$ ) its hedonic value (pleasurable aroma and texture), ii) the strong association between this category of food and craving (Weingarten and Elston, 1990; Rozin et al., 1991; Hill and Heaton-Brown, 1994; Zellner et al., 1999; Rogers and Smit, 2000) - chocolatespecific craving represents almost half of all food cravings (Weingarten and Elston, 1991), and iii) the wellestablished behavioral effects of training IC of responses to chocolate (e.g. Houben and Jansen, 2011, 2015).

For the Aversive group, we selected for each participant five specific vegetable pictures (Aversive NoGo stimuli) and used the same twenty non-chocolate digital colored photographs (Go stimuli) as for the Rewarding group. We used pictures of vegetables as aversive stimuli because of i) their unpleasant value (cf. the picture selection procedure below), and ii) the weak association between this category of food and craving (Rozin and Fallon, 1980). The participants were asked to rate 27 vegetable pictures using a scale from -10 to +10 on the following dimensions: familiarity, emotional response (positive-negative), wanting to eat, taste, consequence to eat (positive-negative). Then, the five images out of 27 possible pictures were chosen as the NoGo stimuli for the aversive group based on each individual participant's lowest rating for taste (scale from -10 (very bad) to +10 (very good)), and in case of equality between the rating of more than 5 pictures, for pleasantness (scale from -10 (negative) to +10 (positive)).

The 20 non-chocolate pictures were the same for the two groups and chosen so that none of them could be encoded in group formation strategies (e.g. tools, office supplies, etc.), with varying colors, and without any semantic link with cooking or food. We included more Go than NoGo items to prevent categorization. All the stimuli were displayed on a white background.

\section{Procedure}

The experiment took place at the laboratory of the Neurology Unit of the University of Fribourg, Switzerland. Participants first gave their written, informed consent, and then completed the questionnaires. Participants' consent was obtained according to the Declaration of Helsinki (BMJ 1991; 302: 1194) and all the procedures approved by our local ethic committee. Participants received either course credits or a monetary compensation for their participation.
Once the electroencephalogram (EEG) system was set up, they sat comfortably in a sound proofed booth at $110 \mathrm{~cm}$ of the 19-inch LCD screen on which stimuli were presented. The Go/NoGo and the Attentional Bias tasks were explained orally before the first block of the task and written instructions were presented again at the beginning of each Attentional Bias and Go/NoGo block.

\section{Go/NoGo task}

In the Go/NoGo paradigm, participants were instructed to press as fast as possible on a button with their right index finger in response to Go stimuli, and to withhold their response to NoGo stimuli. The Go stimuli were all pictures depicting non-food items and the NoGo stimuli were all pictures depicting food items (Fig. 1a).

The Go/NoGo task consisted of 10 blocks of 80 trials each $(50 \% \mathrm{Go} / 50 \% \mathrm{NoGo})$ for a total duration of 50 minutes, with 2-minute breaks in between. A calibration phase of 12 trials (6 Go/6 NoGo) preceded each block. The calibration phase was used to measure the baseline response speed of each participant and to give them a feedback based on this baseline during the subsequent experimental Go/NoGo block. Since a calibration phase preceded each block, the feedback on response speed induced a time pressure and in turn a response prepotency, which was adjusted individually and adapted dynamically across blocks. As a result, the task difficulty was the same across participants and remained stable during the experiment even when participants' performance improved (see Vocat et al., 2008 for similar procedures, Manuel et al., 2010; Hartmann et al., 2016). The baseline response speed was calculated as follow: The mean response time to the 6 Go calibration trials was used as the reaction time threshold (RTt) against which each response times (RT) to Go trials during the experimental blocks were compared. The participants received feedbacks on accuracy and RT: "V" for no response to NoGo trials (correct rejections) and for correct responses to Go trials with RT < 90\%*RTt (fast Hits); "too late" for correct responses to Go trials with $\mathrm{RT}>90 \%{ }^{*} \mathrm{RTt}$ (slow Hits); "X" for button press to NoGo trials (False Alarms: FA) and "no response" for not responding to Go trials (Misses).

Each trial started with the presentation of a black fixation cross at the center of the screen for a duration varying randomly between 950 and 1650 ms. After the offset of the cross, one of the 25 stimuli was presented for $500 \mathrm{~ms}$. Go and NoGo occurrence was equiprobable and their presentation order randomized. At the stimuli onsets, a response window opened for a maximum of $1500 \mathrm{~ms}$, and terminated when the participant responded. The feedback was then presented for $500 \mathrm{~ms}$, and the next trial started. Stimulus presentation and behavioral data recording were controlled with EPrime 2.0 (Psychology Software Tools, Inc., Sharpsburg, PA, USA).

Two measures of behavioral performance were calculated from the Go/NoGo task: the average RT to Go stimuli for each block separately after having 


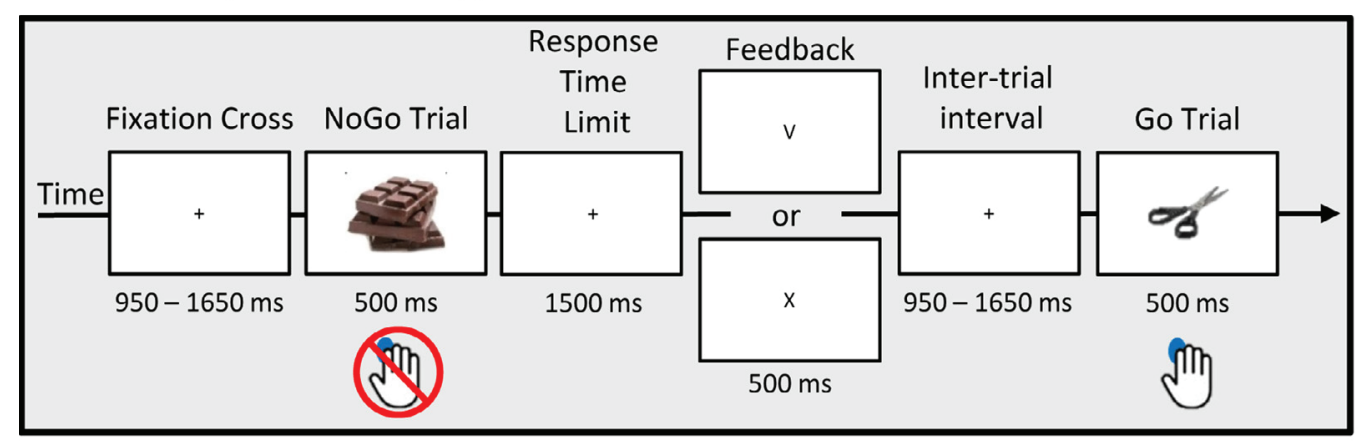

B Attentional Bias Task

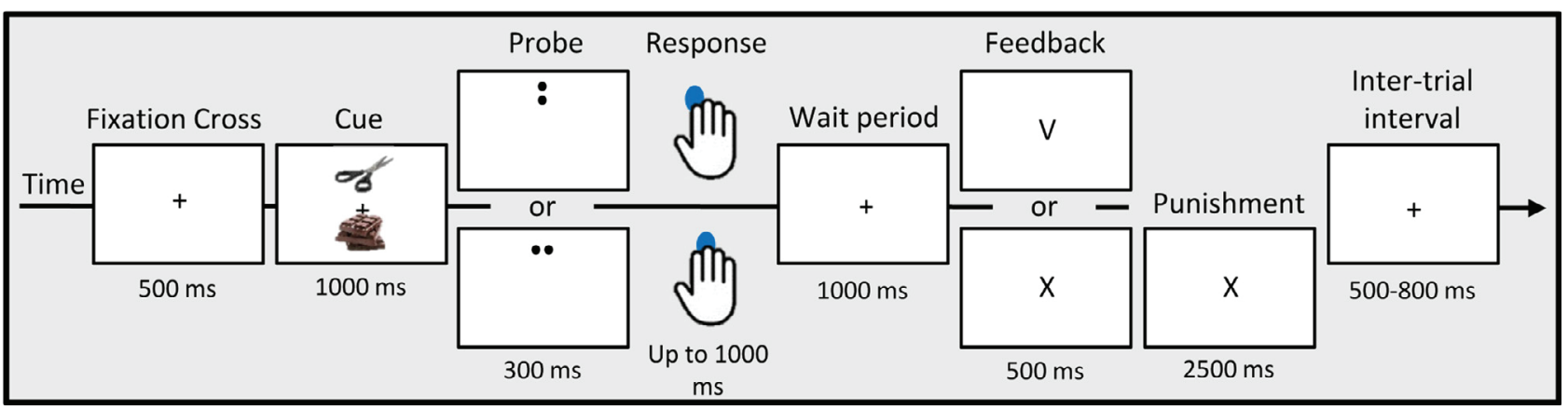

Fig. 1. Schematic representation of the $\mathrm{Go} / \mathrm{NoGo}$ and the attentional bias tasks. (A) Go/NoGo task. Participants had to respond as fast as possible to the Go Trials (Non-food pictures) while withholding their responses to the NoGo stimuli (chocolate in the Reward condition or vegetable pictures in the Aversive condition). (B) Attentional bias Dot-probe task. Pairs of pictures (the cue, i.e. pictures from the Reward Go/NoGo task) were replaced by horizontally or vertically aligned double dots (the probes). The probes were systematically presented at the same (Attend condition) or at the opposite position (Avoid condition) of a given cue. Participants had to discriminate as fast as possible the orientation of the double dots. In both tasks, the ' $V$ ' and ' $X$ ' feedback respectively indicate correct and incorrect responses.

excluded individual RT higher or lower than 2.5 standard deviations from the participant's mean RT; and the percent of errors to NoGo trials (FA rate). On the basis of these two indices, we computed the inverse efficiency score (IES), calculated as the response time to the Go stimuli divided by the percentage of correctly rejected NoGo trials. This index thus takes into account performance to all types of trials during the $\mathrm{Go} / \mathrm{NoGo}$ tasks, and since it is based on both speed and accuracy, it captures the global inhibitory control performance independently on potential speed-accuracy trade-offs. To examine the effects of training, the IES were averaged separately and then statistically compared between the three first blocks (Beginning condition, Beg) and the three last blocks of the training (End condition, End).

\section{Attentional bias task}

We tested if the IC training would decrease attentional biases toward the trained NoGo stimuli by asking the participants to complete a Dot Probe attentional bias task (MacLeod et al., 1986) before and after the Go/NoGo training (Fig. 1b). Participants were presented with cues (the chocolate/vegetable or non-chocolate pictures used as NoGo and Go stimuli during the IC training) immediately replaced by vertically or horizontally aligned double dots probe. The probes were two $1 \mathrm{~mm}$ diameter dots spaced $2 \mathrm{~mm}$ from center. Based on evidence for larger effect sizes when stimuli are displayed in a top-bottom in comparison with side by side presentation (Hakamata et al., 2010), the cues were displayed in a top-bottom configuration. The pre- and post-Go/NoGo training differed only in the order of the cues and the probes.

The attentional bias task included 100 randomly presented trials. Each trial began with a black fixation cross $(500 \mathrm{~ms})$ presented at the center of a white screen. The cross was followed by the cues, one below and one above the fixation cross, during $1000 \mathrm{~ms}$. Then, the probe appeared for $300 \mathrm{~ms}$ at the location of one of the cues. Of the 100 trials, 40 trials had the probe appearing at the same location as the chocolate picture (Attend Rewarding Stimuli Condition), and 40 trials had the probe appearing at the opposite location from the chocolate picture (Avoid Rewarding Stimuli Condition). To decrease the saliency of the task aims, 20 additional trials contained cue pairs with only non-chocolate pictures (Neutral Condition). The location of both the cues and the probes was balanced within each block, as was the horizontal or vertical alignment of the probe dots.

The participants had to respond as fast as possible to the probe by pressing a button during a response window of $1000 \mathrm{~ms}$. They had to press key 1 with the right index finger when the double dots were presented vertically, and press key 2 with the right middle finger when the 
double dots were presented horizontally. Following the participant's response, a blank period of $1000 \mathrm{~ms}$ was presented, followed by a feedback (500 ms).

If the participants responded incorrectly or too slowly (see details on response time thresholds below), they had to wait 2500 ms before the next trial started as a 'punishment'. This extra waiting time aimed to add time pressure. After the feedback a $500-800 \mathrm{~ms}$ inter-trial interval was presented, and the next trial started.

A calibration phase with 10 trials (4 Attend; 4 Avoid; 2 Neutral) preceded the 100 experimental trials. The role and principle of this calibration phase was the same as for the calibration phase during the Go/NoGo task: to determine the reaction time threshold (RTt: average reaction times of the 10 trials), which was used during the experimental phase to add time pressure and individually adjust the difficulty of the task. During the experimentation phase, the feedback depended on the RTt: "V" for correct responses with a RT < RTt; "too late" for correct responses with a RT > RTt; " $X$ " for incorrect responses with RT < RTt; "X and too late" for incorrect response with RT > RTt; and "no response" for omission.

Attentional bias scores were calculated by comparing the average RT to the correctly discriminated probes of both conditions (Attend vs. Avoid). The RT of each trial were averaged after excluding the RT higher and lower than two standard deviations from the individual mean for the pre-Go/NoGo block and the post-Go/NoGo block separately.

\section{EEG recording and preprocessing}

Continuous EEG was recorded with a sampling rate of $1024 \mathrm{~Hz}$ through a 64-channel 10-20 Biosemi ActiveTwo system (Biosemi, Amsterdam, Netherlands), referenced to a ground circuitry (common mode sense/driven right leg ground or CMS-DRL, placed on each side of POz). This circuitry consists of a feedback loop driving the average potential across the montage as close as possible to the amplifier zero (cf. the Biosemi website http://www.biosemi.com/pics/zero_ref1_ big.gif for a diagram).

Offline analyses were performed with the Cartool software (Brunet et al., 2011), and statistical analyses were performed with the RAGU (Koenig et al., 2011) and STEN toolboxes (https://zenodo.org/record/ 1164038).

Before segmenting the raw EEG into epochs, the continuous raw EEG data were filtered with a second order Butterworth with $-12 \mathrm{db} /$ octave roll-off; $0.1 \mathrm{~Hz}$ high-pass, $40 \mathrm{~Hz}$ low-pass; $50 \mathrm{~Hz}$ notch filter. Then, for each participant, we extracted EEG epochs from $100 \mathrm{~ms}$ pre- to $500 \mathrm{~ms}$ post- stimulus onset. Epochs with at least one electrode with at least one time frame at $\pm 80 \mu \mathrm{V}$ were automatically rejected to remove eye blinks and other artifacts. The event-related potential (ERP) averaging was computed separately for each condition, for the first three blocks (Beginning condition, Beg), and the last three blocks of the Go/NoGo training (End condition, End). We focused on the three first and three last blocks as it allowed reaching a reliable signal-to-noise ratio in the ERP while keeping the duration of the training time separating these blocks as long as possible. In addition, this approach ensured comparability with our previous studies in which we applied the same procedure (e.g. Manuel et al., 2010; Manuel et al., 2013; Hartmann et al., 2016). Data from electrode still showing artifacts after the epoch rejection procedure and the ERP averaging were then interpolated using 3D splines (Perrin et al., 1987) (mean \pm SD $=4.1$ $\pm 2.5 \%$ electrodes were interpolated). ERPs were then recalculated to the average reference.

\section{Event-related potentials analyses strategy}

We applied a global, data-driven analyses of the EEG comparing the ERPs between the experimental conditions for all peri-stimulus time frames and across the whole electrode montage. This approach allows minimizing biases and false negative induced by the a priori selection of a limited number of periods and/or electrodes of interest (for corresponding approaches see e.g. Thelen et al., 2012; Hartmann et al., 2016).

Once the period showing modulations in the sensor space were identified, we used it as period of interest for the statistical analyses of the source estimation. We calculated the sources of the ERP for each subject and each condition previously averaged over the period of interest (i.e. showing the ERP modulation). The sources were then statistically compared using the same statistical designs as for the ERP analyses.

\section{ERP analyses}

To identify the effects of training on the Go and NoGo stimuli and between the experimental groups, we computed a NoGo Type (Rewarding; Aversive) $\times$ Stimulus (Go; NoGo) $\times$ Training (Beg; End) mixed ANOVA on the ERP voltages at each electrode for each time frame of the whole ERP time-period. We focused only on the triple interaction term because it ensured that any observed difference reflected the modification that were specific to the NoGo Type (Rewarding vs. Aversive), inhibitory or execution processes (Go vs. NoGo) with training (Beg vs. End).

To correct for multiple comparisons and for temporal and spatial autocorrelation in the EEG data, we took into account only effects with a $p$-value $<0.01$ for at least 11 continuous time points (i.e., here $11 \mathrm{~ms}$ at an EEG sampling rate of $1024 \mathrm{~Hz}$ ) on at least $10 \%$ of the electrodes (Guthrie and Buchwald, 1991).

\section{Electrical source analyses}

The time periods showing ERP NoGo Type (Rewarding; Aversive) $\times$ Stimulus (Go; NoGo) $\times$ Training (Beg; End) interaction were used as periods of interest for the analyses of the electrical source estimations. Brain sources of ERP modulations were estimated using a distributed linear inverse solution model (a minimum norm inverse solution) combined with the local autoregressive average (LAURA) regularization approach, which describes the spatial gradient across 
neighboring solution points (Grave de Peralta Menendez et al., 2001; Grave de Peralta Menendez et al., 2004). LAURA enables investigating multiple simultaneously active sources and selects the configuration of active brain networks which better mimics biophysical behavior of neural fields. In LAURA's approach, the strength of the potentials at a given location depends on the activity of its neighbor nodes according to electromagnetic laws derived from the quasi-static Maxwell's equations stating that the strength of a source falls off with the inverse of the squared distance for potential fields (cubic distance for vector fields; Grave de Peralta Menendez et al., 2001; Grave de Peralta Menendez and Gonzalez Andino, 2002; Grave de Peralta Menendez et al., 2004, for a review see Michel et al., 2004). LAURA uses a realistic head model, and the solution space consists of nodes selected from a grid equally distributed within the gray matter of the Montreal Neurological Institute's average brain (grey matter segmentation courtesy of Grave de Peralta Menendez and Gonzalez Andino; http:// www.electrical-neuroimaging.ch). The head model and lead field matrix were generated with the Spherical Model with Anatomical Constraints (SMAC; Spinelli et al., 2000). As an output, LAURA provides current density measures; their scalar values were evaluated at each node. We used a realistic head model and the solution space included 3005 solution points, selected from a $6 \times 6 \times 6 \mathrm{~mm}$ grid of voxels distributed within the grey matter of the average brain of the Montreal Neurological Institute (MNI, courtesy of R. Grave de Peralta Menendez and S. Gonzalez Andino, University Hospital of Geneva, Geneva, Switzerland). Fundamental and clinical research has assessed the spatial accuracy of this inverse solution and ensure that the reliability of the estimation support the spatial resolution of the interpretation of the localization of the effect in the present study (e.g. Grave de Peralta Menendez et al., 2001; Michel et al., 2004; Gonzalez Andino et al., 2005a; Gonzalez Andino et al., 2005b). The sources estimations were first averaged over the period of interest and the current density at each solution point was subjected to the same NoGo Type $\times$ Stimulus $\times$ Training design as for the ERPs analyses. A spatial correction for multiple tests was achieved by taking into account only clusters showing a p-value $<0.01$ with a spatial-extent criterion $\left(k_{E}\right)$ of $\geq 15$ contiguous nodes. This spatial criterion was determined using the AlphaSim program (available at http://afni.nimh.nih.gov) and assuming a spatial smoothing of $6 \mathrm{~mm}$ full width at half maximum. This program applies a cluster randomization approach. The 10,000 Monte Carlo permutations performed on our lead field matrix revealed a false positive probability of $<0.005$ for a cluster greater than 15 nodes (for a corresponding approach see e.g. De Lucia et al., 2010; Knebel and Murray, 2012; Manuel et al., 2012)

\section{RESULTS}

\section{Behavior}

Go/NoGo task. We analyzed the inverse efficiency score (IES; RT / \% correct NoGo) with a two-way mixed
ANOVA with Training (Beg; End) as a within-subject factor and the NoGo Type (Reward; Aversive) as between-subject factor (Table 1). There was a larger improvement in IC in the Aversive than the Rewarding condition as indexed by a Training $\times$ Stimuli interaction: $F(1,35)=17.812 ; p<0.001 ; \eta_{\mathrm{p}}^{2}=0.337$. There was also a main effect of training $(F(1,35)=35.879$; $\left.p<0.001 ; \eta_{\mathrm{p}}^{2}=0.506\right)$, but no main effect of NoGo Type $\left(F(1,35)=0.811 ; p=0.374 ; \eta_{p}^{2}=0.023\right.$; Fig. $\left.2 a\right)$.

As complementary analyses, we ran the same statistics on the RT and FA rate separately, which suggested that the effect on the IES were mainly driven by changes in RT, as repeatedly observed in previous studies on IC training (e.g. Hartmann et al., 2016; De Pretto et al., 2017): For the RT, there was a Training $\times$ Stimuli interaction: $F(1,35)=17.010 ; p<0.001$; $\left.\eta_{\mathrm{p}}^{2}=0.327\right)$, a main effect of training $(F(1,35)=43.865$; $\left.p<0.001 ; \eta_{\mathrm{p}}^{2}=0.556\right)$, but no main effect of NoGo Type $\left(F(1,35)=0.408 ; p=0.527 ; \eta_{p}^{2}=0.012\right)$. The same analyses were conducted on FA rate, and showed no interaction nor main effect of training (two-way mixed ANOVA Training $\times$ NoGo Type; main effect of Training: $F(1,35)=1.499 ; p=0.229 ; \eta_{\mathrm{p}}^{2}=0.041 ;$ Interaction: $F$ $\left.(1,35)=0.357 ; p=0.554 ; \eta_{\mathrm{p}}^{2}=0.010\right)$, but a small main effect of NoGo Type: $F(1,35)=6.286, \quad p=0.017$; $\eta_{\mathrm{p}}^{2}=0.152$. Of note, the same pattern was found when analyzing median RT instead of mean RTs.

Attentional bias task (Pre- and Post-IC training). We analyzed the attentional biases with a three-way mixed ANOVA with Condition (Avoid; Attend) and Training (Pre-; Post- GNG training) as within-subject factors, and the NoGo Type (Reward; Aversive) as between-subject factor. There was a main effect of Session, driven by a decrease in RT between the pre- and the post- GNG blocks $\left(F(1,35)=114.468 ; \quad p<0.001 ; \quad \eta_{\mathrm{p}}^{2}=0.766\right.$; Fig. 2b), indicating a general, unspecific retest effects. There were no main effect of Condition $(F(1,35)$ $\left.=2.862 ; p=0.100 ; \eta_{p}^{2}=0.076\right)$, and no interactions (Session $\times$ NoGo Type: $F(1,35)=2.713 ; \quad p=0.109$; $\eta_{p}^{2}=0.072$; Condition $\times$ NoGo Type: $F(1,35)=3.193$; $p=0.083 ; \quad \eta_{\mathrm{p}}^{2}=0.084 ; \quad$ Session $\times$ Condition $\times$ NoGo Type: $\quad F(1,35)=1.644 ; \quad p=0.208 ; \quad \eta_{p}^{2}=0.045$ ), indicating that the repeated inhibition to the NoGo stimuli did not influence attentional biases to these stimuli.

\section{Event-related potentials (ERP) and source estimations}

Time-wise electrode-wise ANOVA and source estimation ANOVA. To test for the effect of the IC training to rewarding vs. aversive NoGo items, we applied our Training (Beg; End) by NoGo Type (Reward; Aversive) by Stimulus (Go; NoGo) ANOVA. There was a significant $(p<0.01 ; \quad>10 \mathrm{~ms} ; \quad>10 \%$ electrodes $)$ Training $\times$ NoGo Type $\times$ Stimulus interaction 170 236 ms post-stimulus onset; Fig. 3a,b).

The same NoGo Type $\times$ Training $\times$ Stimulus statistical design was applied on the source estimation previously averaged over the period of significant ERP interaction (Fig. 3c). 
Table 1. Performance at the Go/NoGo tasks and behavioral effects of training

\begin{tabular}{|c|c|c|c|c|c|c|c|}
\hline \multirow{2}{*}{$\begin{array}{l}\text { Mean } \\
\pm S D\end{array}$} & \multicolumn{3}{|c|}{ Reward NoGo } & \multicolumn{3}{|c|}{ Aversive NoGo } & \multirow{2}{*}{$\begin{array}{l}\text { Group } \times \text { Training } \\
\text { Interaction }\end{array}$} \\
\hline & Beginning & End & Beg vs. End & Beginning & End & Beg vs. End & \\
\hline IES & $4.2 \pm 0.4$ & $4.1 \pm 0.6$ & $\begin{array}{l}p=0.213 \\
d_{z}=0.27\end{array}$ & $4.2 \pm 0.3$ & $3.8 \pm 0.2$ & $\begin{array}{l}p<0.001 \\
d_{z}=1.93\end{array}$ & $p<0.001 \eta_{p}^{2}=0.337$ \\
\hline Go RT [ms] & $\begin{array}{l}371.3 \\
\pm 29.9\end{array}$ & $\begin{array}{l}359.3 \\
\pm 45.2\end{array}$ & $\begin{array}{l}p=0.082 \\
d_{z}=0.42\end{array}$ & $\begin{array}{l}399.8 \\
\pm 48.2\end{array}$ & $\begin{array}{l}348.2 \\
\pm 50.2\end{array}$ & $\begin{array}{l}p<0.001 \\
d_{z}=1.74\end{array}$ & $p<0.001 \eta_{p}^{2}=0.327$ \\
\hline $\begin{array}{c}\text { NoGo FA } \\
{[\%]}\end{array}$ & $11.0 \pm 6.3$ & $11.7 \pm 8.9$ & $\begin{array}{l}p=0.656 \\
d_{z}=0.09\end{array}$ & $5.7 \pm 2.8$ & $8.0 \pm 6.8$ & $\begin{array}{l}p=0.212 \\
d_{z}=0.33\end{array}$ & $p=0.554 \eta_{p}^{2}=0.010$ \\
\hline
\end{tabular}

Behavioral performance at the Aversive and Reward Go/NoGo tasks. Mean, SD, as well as the effect size and p-values of comparisons are indicated. IES: Inverse efficiency score; RT: Response time; FA: False alarms.

\section{A Change in response times and false alarm rates (Beg-End) with the Go/NoGo training}

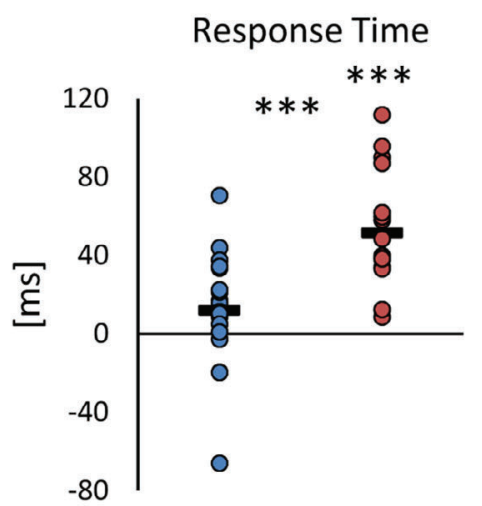

REW AVE
False Alarms

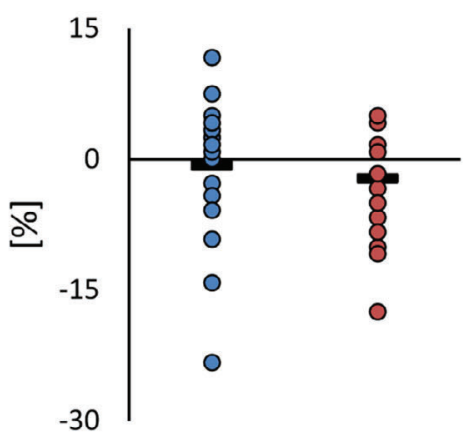

REW

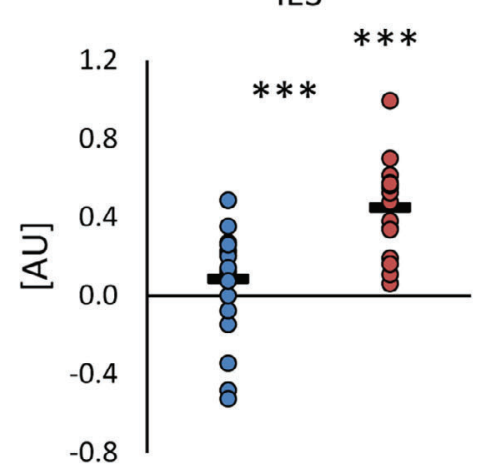

REW AVE

\section{B Change in attentional bias (Pre-Post) to the stimuli used as the NoGo during the training}

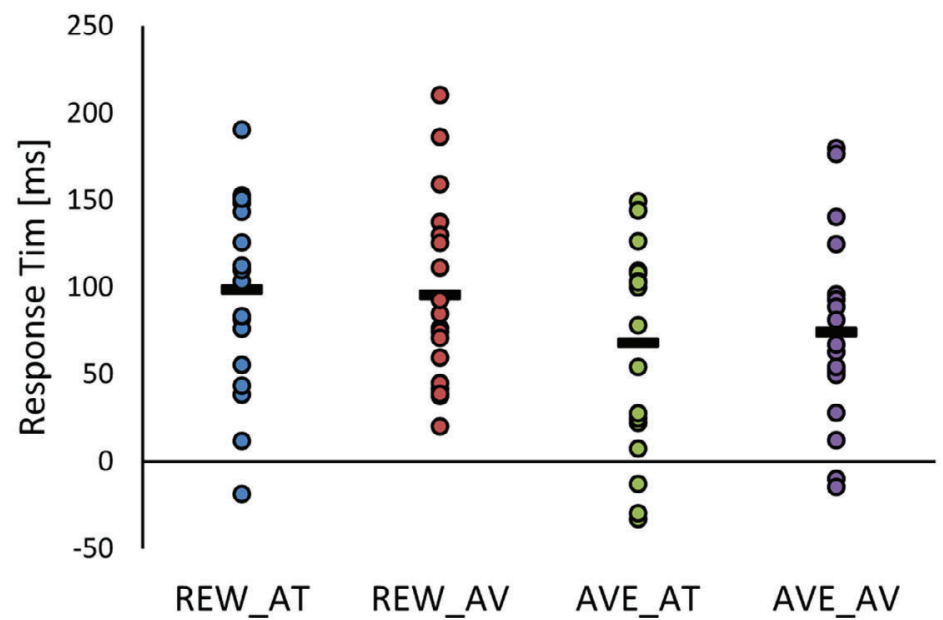

Fig. 2. Behavioral results expressed as the differences before and after training ( $\Delta B$ eg-End in $A, \Delta$ Pre-Post in $B$ ). (A) The difference mean (horizontal line), and individual data (dots) are represented for the response times to Go trials, the difference false alarm rate to NoGo trials, and the combined Inverse Efficiency Score (IES) of the Reward (REW) and Aversive (AVE) Go/NoGo trainings (see Table 1 for the detailed results of the Go/NoGo task). For IES and RT, positive values indicate better scores after the training. For FA negative value indicate better score after the training. (B) The same information is provided for the response time to the probes when they were at the same (Attend, AT) or at the opposite (Avoid, AV) location to the NoGo cue (i.e. the rewarding stimuli used as the NoGo stimuli during the Go/NoGo training, see Table 2 for the detailed results of the Attentional bias task). The Aversive Go/NoGo training improved inhibition performance, as indexed by decreases in response time without concomitant increase of false alarm rate, but did not influence attentional biases to the trained stimuli. Training in the Reward Go/NoGo condition did not result in inhibitory control performance improvement. 
Table 2. Performance at the Aversive and Reward Attentional bias tasks

\begin{tabular}{lllll}
\hline \multirow{2}{*}{ Mean \pm SD } & \multicolumn{2}{l}{ Reward NoGo } & & \multicolumn{2}{c}{ Aversive NoGo } \\
\cline { 2 - 3 } & Pre & Post & Pre & $619.3 \pm 79.3$ \\
\hline Attend & $675.3 \pm 90.9$ & $576.7 \pm 75.1$ & $638.6 \pm 90.4$ & $564.2 \pm 78.4$ \\
Avoid & $673.2 \pm 80.8$ & $577.8 \pm 72.0$ & 94.4 \\
\hline
\end{tabular}

Behavioral performance at the Aversive and Reward Attentional bias tasks. Mean and SD are indicated.

This analysis revealed a significant interaction ( $p<0.05 ; \mathrm{KE} \geq 15$ nodes) within a right orbito-cingulate network extending to the basal ganglia (MNI 10; $50 ;-7$ to $21 ; 14 ;-4)$, and a right temporo-parietal network extending to the precuneus (MNI $51 ;-55 ; 8$ to 26 ; -80 ; 45). The anterior network showed an increase in activity to the rewarding NoGo trials with training and the posterior network an overall decrease in activity. The reverse pattern was observed for the aversive stimuli.

\section{DISCUSSION}

We found that the effect of inhibitory control training interacted with the trained NoGo stimuli reward/aversive value at both the behavioral and electrophysiological levels. We observed larger training-induced performance improvements in the aversive than in the rewarding NoGo stimuli condition. There was, however, no effect of the training on the attentional biases to the stimuli. Neurophysiologically, the training was associated with changes around $200 \mathrm{~ms}$ post-stimulus onset in the response to the NoGo stimuli, driven by an increase in the activity of right orbito-cingulate and a decrease in temporo-parietal areas to the rewarding inhibition stimuli and the reverse pattern to the aversive stimuli. Our findings are most compatible with the associative learning and the behavior-stimulus interaction (BSI) accounts of the effect of inhibitory control training on the behavior toward rewarding stimuli, which respectively posit that repeated motoric inhibitions result in the development of stimulus-driven forms of inhibition and in a devaluation of the stimuli to reduce the conflict between response tendencies and task demands for inhibition.

\section{NoGo stimuli's reward value influences training induced improvements in inhibition performance}

The behavioral effects of training motoric inhibition replicated those reported in previous IC training studies with neutral stimuli, namely a decrease in response times to Go trials with no change in inhibition trials accuracy (Manuel et al., 2010; Benikos et al., 2013; Spierer et al., 2013; Enge et al., 2014; Hartmann et al., 2016). While an improvement in IC would most intuitively manifest as a decrease in commission errors to NoGo trials (i.e. false alarm rate), the present pattern of behavioral change can actually be interpreted as reflecting inhibition enhancement, especially given the time pressure set during the Go/NoGo task: 'horse race' models of inhibition indicate that the IC performance in Go/NoGo and Stopsignal tasks depends on the relative speed of the motor execution and inhibition processes. Hence, a decrease in RT without concomitant change in the rate of false alarms necessarily indicates that the speed of inhibition increased (Chavan et al., 2015; Logan et al., 2014; White et al., 2014; Hartmann et al., 2016). Critically, the effects of training were larger in the Aversive than Reward condition. This finding is compatible with both the 'reinforcement of top-down inhibition' account, which predicted a general improvement for the two NoGo tapes since domain-general process would be improved, and the 'associative learning' account of the effect of training on valuation, which predicted faster learning of the associations between the stopping goals and the aversive pictures because they already elicited withdrawal tendencies. This pattern of results is however inconsistent with the 'Behavior Stimuli Interaction' account, which predicted larger improvement in the aversive than rewarding condition because the aversive pictures were already associated with withdrawal tendencies. Yet, the interaction might also be driven by a dominant effect of response tendencies to rewarding stimuli over inhibition capacities, which would in turn have reduced the effect of training to the rewarding but not to the aversive stimuli.

Regarding the interaction between IC training and attention, while negative results should be interpreted with caution, the absence of effect of the training on attentional biases to the trained NoGo stimuli speaks against an explanation of changes in stimulus valuation in terms of attentional modulations (Houben and Jansen, 2011; Veling and Aarts, 2011; Veling et al., 2013; Wessel et al., 2014; Houben and Jansen, 2015; Wessel et al., 2015). This finding is also in line with previous observation for an absence of interaction between changes in executive control performance and in automatic attentional allocation systems (Sallard et al., 2018). Interactions between inhibitory control and attentional biases might however manifest only during real food choices or consumption, and/or in case of extreme biases or abnormally weak IC (Dawe et al., 2004; Kakoschke et al., 2015). Attentional biases might also be more susceptible to be modified by training decision-related "cognitive" impulsivity than the "motor" impulsivity manipulated in our study (de Wit and Richards, 2004; Olmstead, 2006).

\section{NoGo stimuli's reward value influences training induced changes in the 200 ms latency orbito- cingulate and temporo-parietal activity}

Our effect manifested 170-236 ms post stimulus onset, corroborating most of previous reports on the timing of training-induced changes in IC (180-210 ms in Manuel et al., 2013); 215-240 ms in De Pretto et al., 2017; though effects at 290-400 ms were observed in Hartmann et al., 2016). The $200 \mathrm{~ms}$ latency corresponds to the initiation of 
A Group-averaged ERP Waveforms at two exemplar electrodes
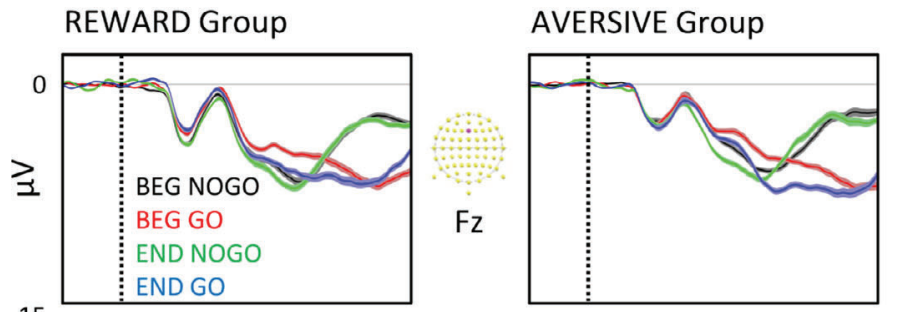

-15
15
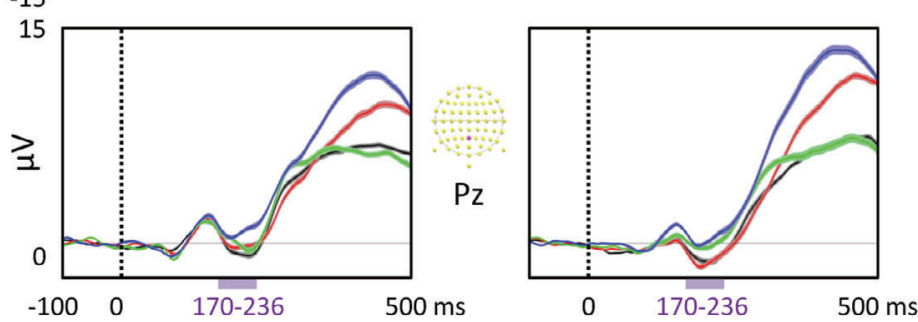

B Time-wise, electrode-wise ERPs ANOVA interaction

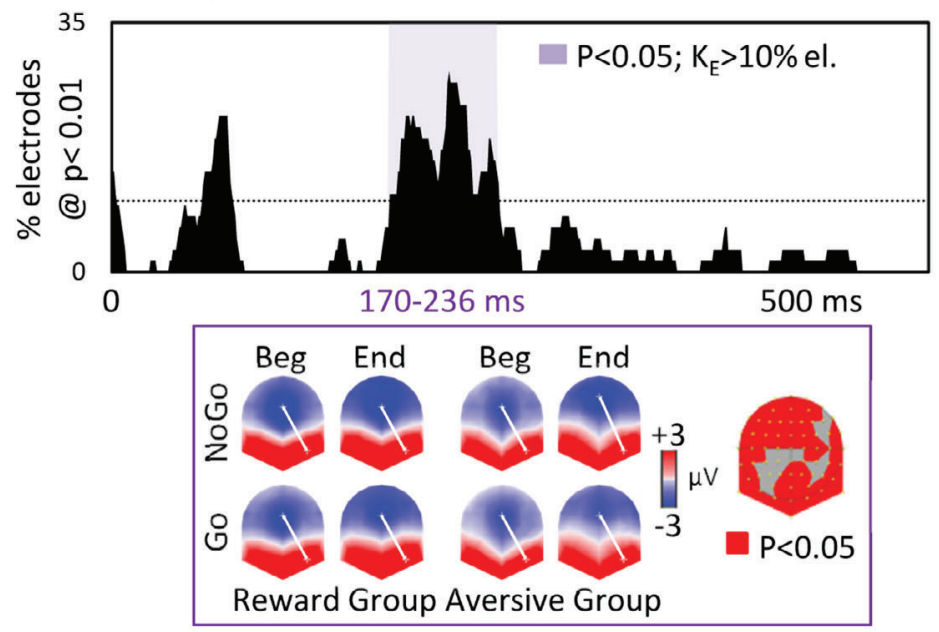

C Distributed source estimations: ANOVA interactions [170-236 ms] $\mathrm{P}<0.05 ; K_{E} \geq 15$
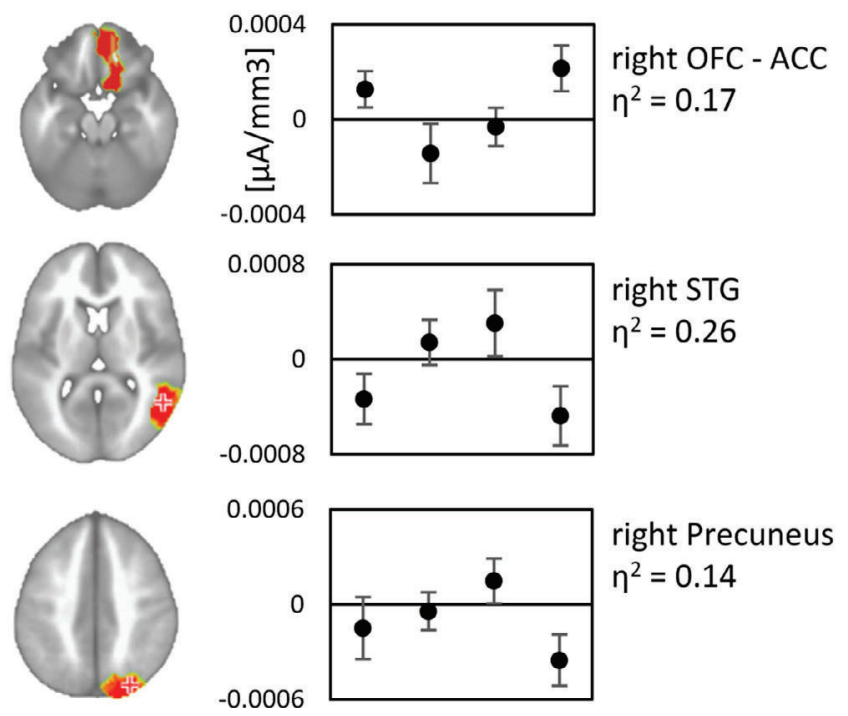

the N2 executive control ERP components (Nieuwenhuis et al., 2003; Kok et al., 2004; Ramautar et al., 2004). In the multi-phase reactive motor inhibition process, this period corresponds to the detection of the conflict between response tendencies and task demands for inhibition (van de Laar et al., 2010; Huster et al., 2013; e.g., Salinas and Stanford, 2013; Logan et al., 2014; Verbruggen et al., 2014a; Verbruggen and Logan, 2015); this phase follows stimulus discrimination (ca 80-100 ms; Salinas and Stanford, 2013; Logan et al., 2014) and the implementation of stimulus-response mapping rules (100-150 ms; Manuel et al., 2010), and precedes the proper implementation of the inhibition command (250-400 ms; Huster et al., 2013).

The latency of our effect during conflict detection and monitoring phases mostly supports the behaviorstimulus interaction (BSI) theory as an account for IC training-induced changes in stimulus responses; Previously observed reductions of the stimuli reward value with IC training may have taken place to decrease the

Fig. 3. Electrical Neuroimaging Results. Interaction terms of the 3-way ANOVAs with the factors: Stimulus (Go; NoGo) $\times$ Training (Beginning; End) $\times$ NoGo Type (Reward NoGo; Aversive NoGo) and Training (Beginning; End). (A) Group-averaged event-related potentials (ERPs) at two exemplar electrodes for the four experimental conditions (ERPs voltage is on the $Y$ axis, time on the $X$ axis, with zero representing the stimulus onset). (B) The result of the ANOVAs' triple interactions for the ERPs at each electrode and each peristimulus time point are represented as the percentage of electrode showing a significant interaction. There was a sustained significant ERP interaction ( $p<0.05$; >11 ms; $>10 \%$ electrodes) around $200 \mathrm{~ms}$ after the onset of the stimuli. The scalp topographies for each condition and the electrodes showing the interaction over this period are represented in red on a flattened EEG cap (nasion upwards). (C) The analyses of the distributed source estimations over the period of significant ERP modulation revealed significant interactions ( $p<0.05$; ke $\geq 15$ nodes) within right orbitofrontal-cingulate (OFC-ACC), right superior temporal (STG), and right precuneus areas. The bar graphs depict the difference (BegEnd) of the mean cluster current densities (interactions' effect size reported). Positive values indicate decrease in activity with training. 
conflict between the inhibition demands and the response tendency to the stimuli (Botvinick et al., 2004; van Veen and Carter, 2002; for a corresponding finding with emotional devaluation, see Kiss et al., 2007). This assumption is also in line with hypotheses that repeated inhibitions modify the motivational significance of the stimuli. In turn, the conflict between the reward-driven response tendency to the stimuli and the response inhibition demand would decrease (Pourtois et al., 2004).

While the latency of the effect speaks against the 'topdown inhibition' account (which predicted effect during later inhibition implementation phases), it does not rule out the 'associative learning' account. Different change in stimulus-response mapping rules would have typically manifested at earlier latencies (Manuel et al., 2010), but they could also have influenced the subsequent behavior-stimulus interaction phase during which our effect manifested.

Further compatible with the BSI and associative learning accounts for the effect of IC training on stimulus valuation, we observed an increase in orbitocingulate activity and a decrease in temporo-parietal activity to the rewarding NoGo stimuli and the reverse pattern for the aversive items. The orbitofrontal cortex is a key node of the reward system involved in encoding the reward values (Kringelbach, 2004, 2005; Rolls and Grabenhorst, 2008), in the rapid reversal of stimulusreinforcement associations (Kringelbach, 2004), and in linking food and other types of rewarding items to hedonic experience (Kringelbach, 2005). The anterior cingulate cortex has been repeatedly involved in conflict monitoring (Carter and van Veen, 2007), notably during the N2 ERP component (Ruggeri et al., 2019). Temporo-parietal areas, notably including the cuneus, show a corresponding functional profile. Activity in these areas are modulated by reward magnitude (Delgado et al., 2003) and by the more automatic signaling of rewarding stimuli saliency (Litt et al., 2011). The direction of our effect suggests an association between higher prefrontal rewardrelated activity with lower-level ability to inhibit motor response tendency, whereas posterior activity would reversely facilitate motor inhibition.

\section{Limitations}

Our study suffers several limitations. First, we did not address whether the observed functional effects would also manifest when the reward value of the stimuli and the associated response tendencies are as prominent as with drugs in addiction (Smith et al., 2014) or food in obesity (Lawrence et al., 2015b). In the same vein, whether corresponding effects of training would take place if other types of rewarding stimuli such as money or smiling faces were used during the training should be investigated in future studies.

Second, while corresponding effects of short and longterm training have been found with neutral stimuli (Chavan et al., 2015; Simonet et al., 2019), we cannot rule out that long-term training might affect differently reward responses.

Finally, we did not test whether our training actually modified the perceived values of the stimuli. Our primary focus was indeed to determine whether the intrinsic reward value of the trained stimuli influenced the neurophysiological effects of training. Hence, we could not conclude if the perceived value of the stimuli changed with the training, only that the value of the stimuli modified the related training-induced changes. Yet, since our training approach was very close to the previous literature demonstrating such effects both in terms of the paradigm and stimuli we used and of the duration of the intervention (Jones et al., 2016; Turton et al., 2016), our training also possibly influenced the perceived value of the stimuli.

Our collective results provide a direct neurophysiological confirmation for an interaction between stimulus rewarding/aversive value and motor inhibitory control training. Our findings further suggest, in line with the predictions from the BSI and associative learning theories, that stimulus devaluation by repeated motoric inhibition observed in previous food IC training studies most likely followed from modulations at the level of the conflict between reward-driven response tendencies and task demands for inhibition. Our study also extends current neurocognitive models of traininginduced IC plasticity by showing that the nature of the stimuli to which responses have to be inhibited during the training directly influences its functional effects.

\section{ACKNOWLEDGMENTS}

We thank Dr Michael Mouthon and Sonia Pupillo for their help in data collection, and Dr Ulrike Toepel and Prof. Simone Munsch for discussions on earlier versions of the manuscript. This work was supported by a grant from the Swiss National Science Foundation, Switzerland (Grants \#316030_144998 and \#320030_175469 to LS). The Cartool software (brainmap ping.unige.ch/cartool) has been programmed by Denis Brunet, from the Functional Brain Mapping Laboratory, Geneva, Switzerland, and is supported by the Center for Biomedical Imaging (CIBM) of Geneva and Lausanne. The STEN toolbox (http://doi.org/10.5281/zenodo. 1164038) has been programmed by Jean-François Knebel and Michael Notter, from the Laboratory for Investigative Neurophysiology (the LINE), Lausanne, Switzerland, and is supported by the Center for Biomedical Imaging (CIBM) of Geneva and Lausanne, Switzerland, and by National Center of Competence in Research project "SYNAPSY - The Synaptic Bases of Mental Disease", Switzerland; project no. 51AU40_125759. Dr. Garcia-Burgos' current affiliation is the Department of Psychobiology, Institute of Neurosciences, University of Granada, 18071, Granada, Spain.

\section{REFERENCES}

Allom V, Mullan B, Hagger M (2016) Does inhibitory control training improve health behaviour? A meta-analysis. Health Psychol Rev 10:168-186.

Anderson BA, Laurent PA, Yantis S (2013) Reward predictions bias attentional selection. Front Hum Neurosci 11(7):262.

Aron AR, Robbins TW, Poldrack RA (2004) Inhibition and the right inferior frontal cortex. Trends Cogn Sci 8:170-177. 
Benikos N, Johnstone SJ, Roodenrys SJ (2013) Short-term training in the Go/Nogo task: behavioural and neural changes depend on task demands. Int J Psychophysiol 87:301-312.

Benton D, Greenfield K, Morgan M (1998) The development of the attitudes to chocolate questionnaire. Personality Individ Differ 24:513-520.

Berkman ET, Kahn LE, Merchant JS (2014) Training-induced changes in inhibitory control network activity. J Neurosci 34:149-157.

Blechert J, Meule A, Busch NA, Ohla K (2014) Food-pics: an image database for experimental research on eating and appetite. Front Psychol 5:617.

Botvinick MM, Cohen JD, Carter CS (2004) Conflict monitoring and anterior cingulate cortex: an update. Trends Cogn Sci 8:539-546.

Brunet D, Murray MM, Michel CM (2011) Spatiotemporal analysis of multichannel EEG: CARTOOL. Comput Intell Neurosci 2011 813870.

Carter CS, van Veen V (2007) Anterior cingulate cortex and conflict detection: an update of theory and data. Cogn Affect Behav Neurosci 7:367-379.

Chavan CF, Mouthon M, Draganski B, van der Zwaag W, Spierer L (2015) Differential patterns of functional and structural plasticity within and between inferior frontal gyri support training-induced improvements in inhibitory control proficiency. Hum Brain Mapp 36:2527-2543.

Chen Z, Veling H, Dijksterhuis A, Holland RW (2016) How does not responding to appetitive stimuli cause devaluation: evaluative conditioning or response inhibition? J Exp Psychol Gen 145:1687-1701.

Dawe S, Gullo MJ, Loxton NJ (2004) Reward drive and rash impulsiveness as dimensions of impulsivity: implications for substance misuse. Addict Behav 29:1389-1405.

De Lucia M, Clarke S, Murray MM (2010) A temporal hierarchy for conspecific vocalization discrimination in humans. J Neurosci 30:11210-11221.

De Pretto M, Rochat L, Spierer L (2017) Spatiotemporal brain dynamics supporting the immediate automatization of inhibitory control by implementation intentions. Sci Rep 7:10821.

de Wit H, Richards JB (2004) Dual determinants of drug use in humans: reward and impulsivity. Nebraska Symposium on Motivation Nebraska Symposium on Motivation 50:19-55.

Delgado MR, Locke HM, Stenger VA, Fiez JA (2003) Dorsal striatum responses to reward and punishment: effects of valence and magnitude manipulations. Cogn, Affective Behav Neurosci 3:27-38.

Enge S, Behnke A, Fleischhauer M, Kuttler L, Kliegel M, Strobel A (2014) No evidence for true training and transfer effects after inhibitory control training in young healthy adults. J Exp Psychol Learn Mem Cogn 40:987-1001.

Falkenstein M (2006) Inhibition, conflict and the Nogo-N2. Clin Neurophysiol 117:1638-1640.

Gonzalez Andino SL, Michel CM, Thut G, Landis T, Grave de Peralta $\mathrm{R}$ (2005a) Prediction of response speed by anticipatory highfrequency (gamma band) oscillations in the human brain. Hum Brain Mapp 24:50-58.

Gonzalez Andino SL, Murray MM, Foxe JJ, de Peralta Menendez RG (2005b) How single-trial electrical neuroimaging contributes to multisensory research. Exp Brain Res 166:298-304.

Goolsby BA, Shapiro KL, Raymond JE (2009) Distractor devaluation requires visual working memory. Psychon Bull Rev 16:133-138.

Grave de Peralta Menendez R, Gonzalez Andino S, Lantz G, Michel CM, Landis T (2001) Noninvasive localization of electromagnetic epileptic activity. I. Method descriptions and simulations. Brain Topogr 14:131-137.

Grave de Peralta Menendez R, Gonzalez Andino SL (2002) Comparison of algorithms for the localization of focal sources: Evaluation with simulated data and analysis of experimental data. Int J Bioelectromagn 4.
Grave de Peralta Menendez R, Murray MM, Michel CM, Martuzzi R, Gonzalez Andino SL (2004) Electrical neuroimaging based on biophysical constraints. Neurolmage 21:527-539.

Guthrie D, Buchwald JS (1991) Significance testing of difference potentials. Psychophysiology 28:240-244.

Hakamata Y, Lissek S, Bar-Haim Y, Britton JC, Fox NA, Leibenluft E, Ernst M, Pine DS (2010) Attention bias modification treatment: a meta-analysis toward the establishment of novel treatment for anxiety. Biol Psychiatry 68:982-990.

Hartmann L, Sallard E, Spierer L (2016) Enhancing frontal top-down inhibitory control with Go/NoGo training. Brain Struct Funct.

Herman CP, Polivy J (1980) Restrained eating. Obesity (Silver Spring):208-225

Hill AJ, Heaton-Brown L (1994) The experience of food craving: a prospective investigation in healthy women. J Psychosom Res 38:801-814.

Houben K, Jansen A (2011) Training inhibitory control. A recipe for resisting sweet temptations. Appetite 56:345-349.

Houben K, Jansen A (2015) Chocolate equals stop. Chocolatespecific inhibition training reduces chocolate intake and go associations with chocolate. Appetite 87:318-323.

Huster RJ, Enriquez-Geppert S, Lavallee CF, Falkenstein M, Herrmann CS (2013) Electroencephalography of response inhibition tasks: functional networks and cognitive contributions. Int J Psychophysiol 87:217-233.

Jasinska AJ, Yasuda M, Burant CF, Gregor N, Khatri S, Sweet M, Falk EB (2012) Impulsivity and inhibitory control deficits are associated with unhealthy eating in young adults. Appetite 59:738-747.

Jones A, Di Lemma LC, Robinson E, Christiansen P, Nolan S, TudurSmith C, Field M (2016) Inhibitory control training for appetitive behaviour change: a meta-analytic investigation of mechanisms of action and moderators of effectiveness. Appetite 97:16-28.

Kakoschke N, Kemps E, Tiggemann M (2015) Combined effects of cognitive bias for food cues and poor inhibitory control on unhealthy food intake. Appetite 87:358-364.

Kiss M, Goolsby BA, Raymond JE, Shapiro KL, Silvert L, Nobre AC, Fragopanagos N, Taylor JG, Eimer M (2007) Efficient attentional selection predicts distractor devaluation: event-related potential evidence for a direct link between attention and emotion. J Cogn Neurosci 19:1316-1322.

Knebel JF, Murray MM (2012) Towards a resolution of conflicting models of illusory contour processing in humans. Neurolmage 59:2808-2817.

Koenig T, Kottlow M, Stein M, Melie-Garcia L (2011) Ragu: a free tool for the analysis of EEG and MEG event-related scalp field data using global randomization statistics. Comput Intell Neurosci 2011 938925.

Kok A, Ramautar JR, De Ruiter MB, Band GP, Ridderinkhof KR (2004) ERP components associated with successful and unsuccessful stopping in a stop-signal task. Psychophysiology 41:9-20.

Kringelbach ML (2004) Food for thought: hedonic experience beyond homeostasis in the human brain. Neuroscience 126:807-819.

Kringelbach ML (2005) The human orbitofrontal cortex: linking reward to hedonic experience. Nat Rev Neurosci 6:691-702.

Kringelbach ML, Rolls ET (2004) The functional neuroanatomy of the human orbitofrontal cortex: evidence from neuroimaging and neuropsychology. Prog Neurobiol 72:341-372.

Lawrence NS, O'Sullivan J, Parslow D, Javaid M, Adams RC, Chambers CD, Kos K, Verbruggen F (2015a) Training response inhibition to food is associated with weight loss and reduced energy intake. Appetite 95:17-28.

Lawrence NS, Verbruggen F, Morrison S, Adams RC, Chambers CD (2015b) Stopping to food can reduce intake. Effects of stimulusspecificity and individual differences in dietary restraint. Appetite 85:91-103.

Lenartowicz A, Verbruggen F, Logan GD, Poldrack RA (2011) Inhibition-related activation in the right inferior frontal 
gyrus in the absence of inhibitory cues. J Cogn Neurosci 23:3388-3399.

Litt A, Plassmann H, Shiv B, Rangel A (2011) Dissociating valuation and saliency signals during decision-making. Cereb Cortex 21:95-102.

Loeber S, Grosshans M, Herpertz S, Kiefer F, Herpertz SC (2013) Hunger modulates behavioral disinhibition and attention allocation to food-associated cues in normal-weight controls. Appetite 71:32-39.

Logan GD, Van Zandt T, Verbruggen F, Wagenmakers EJ (2014) On the ability to inhibit thought and action: general and special theories of an act of control. Psychol Rev 121:66-95.

MacLeod C, Mathews A, Tata P (1986) Attentional bias in emotional disorders. J Abnorm Psychol 95:15-20.

Manuel AL, Bernasconi F, Murray MM, Spierer L (2012) Spatiotemporal brain dynamics mediating post-error behavioral adjustments. J Cogn Neurosci 24:1331-1343.

Manuel AL, Bernasconi F, Spierer L (2013) Plastic modifications within inhibitory control networks induced by practicing a stopsignal task: an electrical neuroimaging study. Cortex 49:1141-1147.

Manuel AL, Grivel J, Bernasconi F, Murray MM, Spierer L (2010) Brain dynamics underlying training-induced improvement in suppressing inappropriate action. J Neurosci 30:13670-13678.

Meule A, Lutz AP, Krawietz V, Stutzer J, Vogele C, Kubler A (2014) Food-cue affected motor response inhibition and self-reported dieting success: a pictorial affective shifting task. Front Psychol 5:216.

Meule A, Platte P (2016) Attentional bias toward high-calorie foodcues and trait motor impulsivity interactively predict weight gain. Health Psychol Open 3.

Michel CM, Murray MM, Lantz G, Gonzalez S, Spinelli L, Grave de Peralta R (2004) EEG source imaging. Clin Neurophysiol 115:2195-2222.

Nederkoorn C, Guerrieri R, Havermans RC, Roefs A, Jansen A (2009) The interactive effect of hunger and impulsivity on food intake and purchase in a virtual supermarket. Int J Obes (Lond) 33:905-912.

Nieuwenhuis S, Yeung N, van den Wildenberg W, Ridderinkhof KR (2003) Electrophysiological correlates of anterior cingulate function in a go/no-go task: effects of response conflict and trial type frequency. Cogn, Affective Behav Neurosci 3:17-26.

Nijs IM, Muris P, Euser AS, Franken IH (2010) Differences in attention to food and food intake between overweight/obese and normal-weight females under conditions of hunger and satiety. Appetite 54:243-254.

Olmstead MC (2006) Animal models of drug addiction: Where do we go from here? Q J Exp Psychol 59:625-653.

Patton JH, Stanford MS, Barratt ES (1995) Factor structure of the Barratt impulsiveness scale. J Clin Psychol 6:768-774.

Perrin F, Pernier J, Bertrand O, Giard MH, Echallier JF (1987) Mapping of scalp potentials by surface spline interpolation. Electroencephalogr Clin Neurophysiol 66:75-81.

Pourtois G, Grandjean D, Sander D, Vuilleumier P (2004) Electrophysiological correlates of rapid spatial orienting towards fearful faces. Cereb Cortex 14:619-633.

Ramautar JR, Kok A, Ridderinkhof KR (2004) Effects of stop-signal probability in the stop-signal paradigm: the N2/P3 complex further validated. Brain Cogn 56:234-252.

Rogers PJ, Smit HJ (2000) Food craving and food "addiction": a critical review of the evidence from a biopsychosocial perspective. Pharmacol Biochem Behav 66:3-14.

Rolls ET, Grabenhorst F (2008) The orbitofrontal cortex and beyond: from affect to decision-making. Prog Neurobiol 86:216-244.

Rozin P, Levine E, Stoess C (1991) Chocolate craving and liking. Appetite 17:199-212.

Rozin P, Fallon A (1980) The psychological categorization of foods and non-foods: A preliminary taxonomy of food rejection. Appetite 1:193-201.
Salinas E, Stanford TR (2013) The countermanding task revisited: fast stimulus detection is a key determinant of psychophysical performance. J Neurosci 33:5668-5685.

Sallard E, Hartmann L, Ptak R, Spierer L (2018) Spatiotemporal brain dynamics underlying attentional bias modifications. Int $\mathrm{J}$ Psychophysiol 130:29-39.

Simonet M, Roten FCV, Spierer L, Barral J (2019) Executive control training does not generalize, even when associated with plastic changes in domain-general prefrontal areas. Neuroimage. 15 (197):457-469. neuroimage.2019.04.010. Epub 2019 Apr 8.

Smith JL, Mattick RP, Jamadar SD, Iredale JM (2014) Deficits in behavioural inhibition in substance abuse and addiction: a metaanalysis. Drug Alcohol Depend 145:1-33.

Spierer L, Chavan CF, Manuel AL (2013) Training-induced behavioral and brain plasticity in inhibitory control. Front Hum Neurosci 7:427.

Spinelli L, Andino SG, Lantz G, Seeck M, Michel CM (2000) Electromagnetic inverse solutions in anatomically constrained spherical head models. Brain Topogr 13:115-125.

Stice E, Lawrence NS, Kemps E, Veling H (2016) Training motor responses to food: A novel treatment for obesity targeting implicit processes. Clin Psychol Rev. 49:16-27. https://doi.org/10.1016/j. cpr.2016.06.005. Epub 2016 Jul 21.

Thelen A, Cappe C, Murray MM (2012) Electrical neuroimaging of memory discrimination based on single-trial multisensory learning. Neurolmage 62:1478-1488.

Turton R, Bruidegom K, Cardi V, Hirsch CR, Treasure J (2016) Novel methods to help develop healthier eating habits for eating and weight disorders: a systematic review and meta-analysis. Neurosci Biobehav Rev 61:132-155.

van de Laar MC, van den Wildenberg WP, van Boxtel GJ, van der Molen MW (2010) Processing of global and selective stop signals: application of Donders' subtraction method to stop-signal task performance. Exp Psychol 57:149-159.

van Veen V, Carter CS (2002) The anterior cingulate as a conflict monitor: fMRI and ERP studies. Physiol Behav. 77(4-5):477-482. Review.

Veling $H$, Aarts $H$ (2011) Changing impulsive determinants of unhealthy behaviours towards rewarding objects. Health Psychology Review 5:150-153.

Veling H, Aarts H, Stroebe W (2013) Stop signals decrease choices for palatable foods through decreased food evaluation. Front Psychol 4:875.

Veling H, Holland RW, van Knippenberg A (2008) When approach motivation and behavioral inhibition collide: behavior regulation through stimulus devaluation. J Exp Soc Psychol 44:1013-1019.

Veling $\mathrm{H}$, Lawrence NS, Chen Z, van Koningsbruggen GM, Holland RW (2017) What is trained during food Go/No-Go training? A review focusing on mechanisms and a research Agenda. Curr Addict Rep 4:35-41.

Verbruggen F, Best M, Bowditch WA, Stevens T, McLaren IP (2014a) The inhibitory control reflex. Neuropsychologia 65:263-278.

Verbruggen F, Logan GD (2015) Evidence for capacity sharing when stopping. Cognition 142:81-95.

Verbruggen F, Stevens T, Chambers CD (2014b) Proactive and reactive stopping when distracted: an attentional account. J Exp Psychol Hum Percept Perform 40:1295-1300.

Vocat R, Pourtois G, Vuilleumier P (2008) Unavoidable errors: a spatio-temporal analysis of time-course and neural sources of evoked potentials associated with error processing in a speeded task. Neuropsychologia 46:2545-2555.

Weingarten HP, Elston D (1990) The phenomenology of food cravings. Appetite 15:231-246.

Weingarten HP, Elston D (1991) Food cravings in a college population. Appetite 17:167-175.

Wessel JR, O'Doherty JP, Berkebile MM, Linderman D, Aron AR (2014) Stimulus devaluation induced by stopping action. J Exp Psychol Gen 143:2316-2329. 
Wessel JR, Tonnesen AL, Aron AR (2015) Stimulus devaluation induced by action stopping is greater for explicit value representations. Front Psychol 6:1640.

White CN, Congdon E, Mumford JA, Karlsgodt KH, Sabb FW, Freimer NB, London ED, Cannon TD, Bilder RM, Poldrack RA (2014) Decomposing decision components in the stop-signal task: a model-based approach to individual differences in inhibitory control. J Cogn Neurosci 26:1601-1614.

Zellner DA, Garriga-Trillo A, Rohm E, Centeno S, Parker S (1999) Food

liking and craving: a cross-cultural approach. Appetite 33:61-70.

Zigmond AS, Snaith RP (1983) The hospital anxiety and depression scale. Acta Psychiatr Scand 67:361-370. 